\title{
Fast-time variability in high-mass X-ray binaries: SAX J2103.5+4545
}

\section{Pablo Reig*}

IESL, Foundation for Reasearch \& Technology-Hellas, Heraklion, Crete GR - 711 10, Greece E-mail: pauephysics.uoc.gr

\section{Agnieszka Słowikowska}

Institute of Astronomy, University of Zielona Góra, Lubuska 2, 65-265 Zielona Góra, Poland E-mail: aga@physics.uoc.gr

\section{Gottfried Kanbach}

Max Planck Institute for Extraterrestrial Physics, Giessenbachstrasse 1, 85741 Garching,

Germany

E-mail: gok@mpe.mpg.de

We present OPTIMA observations of the high-mass X-ray binary SAX J2103.5+4545 covering various orbital phases. Our aim is to investigate the fast optical variability (down to $\sim 0.1 \mathrm{~s}$ ) as a function of the orbital phase in an attempt to search for signatures of the interaction of the neutron star with the Be circumstellar disc. The background and flat-field corrected OPTIMA light curves were used to create power spectra. The spectral continuum is well represented by a power law. We find a correlation between the power-law index and the fractional amplitude of variability with the orbital phase. We speculate that this correlation might be caused by the disruption of the disc by the close passage of the neutron star.

High Time Resolution Astrophysics IV - The Era of Extremely Large Telescopes - HTRA-IV,

May 5-7, 2010

Agios Nikolaos, Crete, Greece

\footnotetext{
* Speaker.
} 


\begin{tabular}{lccccccc}
\hline Date & $2,400,000+$ & $\begin{array}{c}\text { Exposure } \\
\text { time }(\mathrm{s})\end{array}$ & $\begin{array}{c}<I>^{a} \\
\mathrm{cs}^{-1}\end{array}$ & $\Gamma$ & $\begin{array}{c}r m s \\
(\%)\end{array}$ & $\begin{array}{c}\text { Proj(M-S })^{b} \\
\left({ }^{\circ}\right)\end{array}$ & $\begin{array}{c}\text { Moon }^{c} \\
\text { illum. }(\%)\end{array}$ \\
\hline $11-07-2006$ & 53927.959 & 4116 & 21.3 & $1.20 \pm 0.03$ & $15 \pm 4$ & 71 & 99 \\
$08-09-2008$ & 54717.949 & 1400 & 24.6 & $1.21 \pm 0.02$ & $26 \pm 6$ & 84 & 63 \\
$11-09-2008$ & 54720.804 & 3390 & 25.6 & $0.50 \pm 0.02$ & $10 \pm 2$ & 66 & 86 \\
$03-10-2008$ & 54742.919 & 2400 & 20.5 & $1.04 \pm 0.01$ & $30 \pm 5$ & 99 & 20 \\
$29-10-2008$ & 54768.869 & 3150 & 20.6 & $1.15 \pm 0.01$ & $26 \pm 5$ & 107 & 1 \\
$04-11-2008$ & 54774.828 & 3060 & 23.5 & $0.52 \pm 0.02$ & $11 \pm 2$ & 64 & 37 \\
$05-11-2008$ & 54775.841 & 4000 & 23.1 & $1.17 \pm 0.02$ & $19 \pm 4$ & 64 & 47 \\
\hline
\end{tabular}

$a: \times 10^{3}$ background subtracted intensity

$b$ : Distance between the source and the Moon (in degrees) projected on the sky

$c$ : Moon illumination: Full Moon $=100 \%$, New Moon $=0 \%$

Table 1: OPTIMA observations of SAX J2103.5+4545.

\section{Introduction}

We are currently monitoring a number of high-mass X-ray binaries (HMXBs) with the Optical Pulsar TIMing Analyzer (OPTIMA) with the aim of investigating whether the compact companion has any observational effect on the circumstellar disc of the Be star companion. We observe the sources at different orbital phases and study any possible difference. Especially relevant are the observations taken during periastron and apastron. We sample time variability down to $0.1 \mathrm{~s}$.

OPTIMA is a high-time resolution optical photo-polarimeter [1] that was designed to measure optical light curves of highly variable binary systems in order to cross-correlate and to establish timing relations to emissions in other wavelength ranges (radio and X-rays).

HMXB consist of a neutron star orbiting around an early-type star. They divide into two major groups according to the luminosity class of the optical component: supergiant X-ray binaries if the massive companion is an evolve star (luminosity class I-II) and Be/X-ray binaries (BeX) if it is a main-sequence or giant star. SAX J2103.5+4545 is an unusual HMXB. Its spin, $P_{\text {spin }}=358.6 \mathrm{~s}$ and orbital, $P_{\text {orb }}=12.7 \mathrm{~d}$ periods are typical of supergiant X-ray binaries. In fact, falls in the wind-fed supergiant region of the $P_{o r b}-P_{\text {spin }}$ diagram. However, the primary component of the binary is a B0V [2].

\section{Observations}

OPTIMA observations of SAX J2103.5+4545 were made from the Skinakas observatory (Crete, Greece) during the summer 2008. An older observation made in July 2006 was also included. Table 1 shows the log of the OPTIMA observations. 


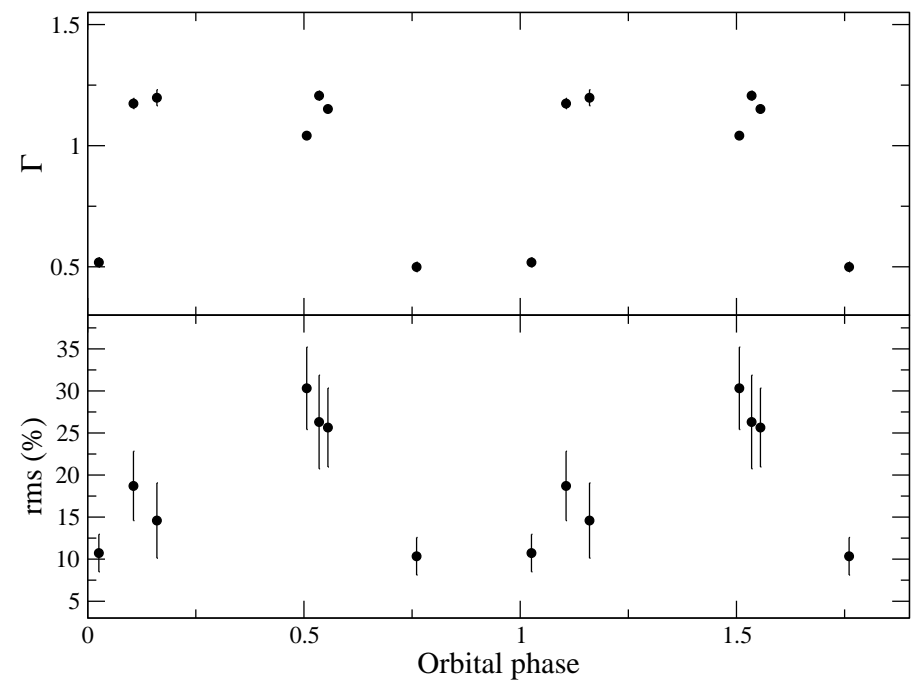

Figure 1: Power-law index and $0.01-1 \mathrm{~Hz} r m s$ variability as a function of orbital phase.

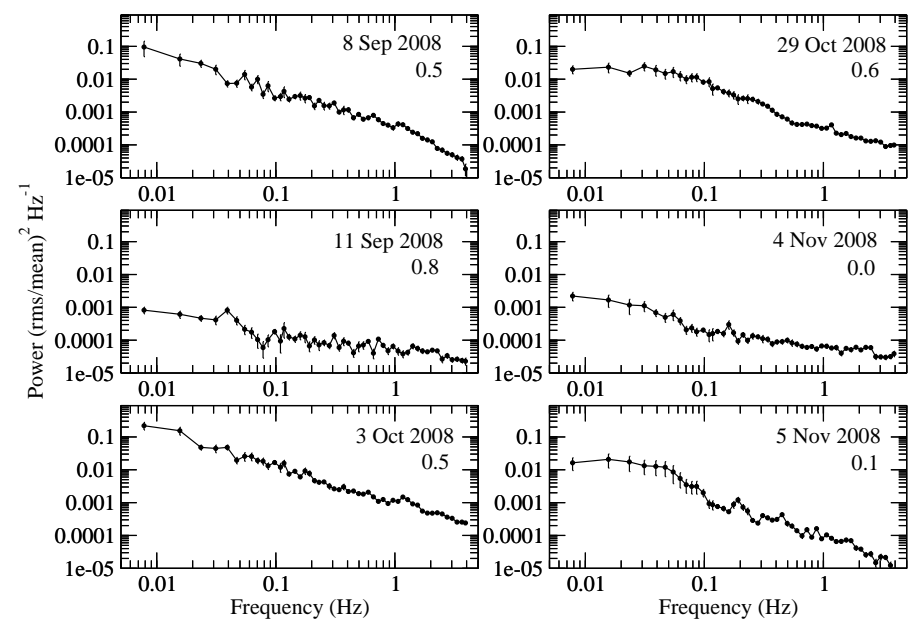

Figure 2: OPTIMA power spectra of SAX J2103.5+4545. The number below the date is the orbital phase.

\section{Results}

The source was observed with OPTIMA at different instances of its orbit. The OPTIMA light curve was divided into 128-s segments with a resolution of $0.125 \mathrm{~s}$ and an FFT was obtained for each segment. The final power spectrum resulted after averaging all the individual power spectra and rebinning logarithmically in frequency. In general, the OPTIMA power spectra fit well to a single power law. The fourth and fifth columns in Table 1 give the best-fit power-law index and the fraction of variability in the frequency range $0.01-1 \mathrm{~Hz}$, respectively, while Fig. 1 shows these two quantities as a function of orbital phase. At around periastron passage (orbital phase 0.4), the $0.01-1 \mathrm{~Hz}$ fractional amplitude of variability, rms is maximum while the power-law index is minimum. Various representative power spectra are shown in Fig. 国. 


\section{Discussion}

Reig et al. (2010) showed that SAX J2103.5+4545 exhibits the fastest time scales for the disappearance and reformation of the circumstellar disc, compared to other typical Be/X-ray binaries. In less than 2 years, SAX J2103.5+4545 is capable to form and lose the disc. They attributed this behaviour to the narrow orbit of the system. The neutron star truncates the disc and prevents it from extending to large radii. Since the source was not X-ray active at the time of the OPTIMA observations, X-ray reprocessing can be ruled out as the mechanism responsible for the increase in the amplitude of variability at 1-s timescales during periastron. Instead it might be caused by the interaction of the neutron star with the Be star's disc. New OPTIMA observations of this and other high-mass X-ray binaries will clarify this issue.

\section{Acknowledgments}

A. Słowikowska acknowledges financial support from the Foundation for Polish Science grant HOM/2009/11B and the Marie Curie European Reintegration Grant PERG05-GA-2009-249168.

\section{References}

[1] Kanbach et al., The OPTIMA photo-polarimeter: updated configuration and calibration, 2010, see contribution in this volume

[2] P. Reig, A. Słowikowska, A. Zezas \& P. Blay, Correlated optical/X-ray variability in the high-mass $X$-ray binary SAX J2103.5+4545, MNRAS 401, pp. 55-66, 2010, [arXiv: 0908 . 4 497v1] 